\title{
Correction to: Deposition of bismuth sulfide and aluminum doped bismuth sulfide thin films for photovoltaic applications
}

\author{
, Tanzeela Fazal ${ }^{1}$, Shahid Iqbal ${ }^{2, *}$ (D), Mazloom Shah ${ }^{1}$, Ali Bahadur ${ }^{3, \star}$, Bushra Ismail ${ }^{4}$, \\ Hisham S. M. Abd-Rabboh ${ }^{5,6}$, Rabia Hameed ${ }^{1}$, Qaiser Mahmood ${ }^{7, *}$, Aliya Ibrar ${ }^{8}$, \\ Muhammad Sufyan Nasar ${ }^{2}$, Yasir Ehsan ${ }^{2}$, Ahmad Nauman Shah Saqib ${ }^{9}$, and \\ Adnan $^{10}$ \\ ${ }^{1}$ Department of Chemistry, Abbottabad University of Science and Technology (AUST), Abbottabad, Pakistan \\ ${ }^{2}$ Department of Chemistry, School of Natural Sciences (SNS), National University of Science and Technology (NUST), \\ $\mathrm{H}-12$, Islamabad 46000, Pakistan \\ ${ }^{3}$ Department of Transdisciplinary Studies, Graduate School of Convergence Science and Technology, Seoul National University, \\ Seoul 08826, South Korea \\ ${ }^{4}$ Department of Chemistry, COMSATS University Islamabad (CUI), Abbottabad Campus, Abbottabad 22060, Pakistan \\ ${ }^{5}$ Chemistry Department, Faculty of Science, King Khalid University, P.O. Box 9004, Abha 61413, Saudi Arabia \\ ${ }^{6}$ Department of Chemistry, Faculty of Science, Ain Shams University, Abbassia 11566, Cairo, Egypt \\ ${ }^{7}$ Chemistry and Chemical Engineering Guangdong Laboratory, Shantou 515031, People's Republic of China \\ ${ }^{8}$ Department of Chemistry, Faculty of Natural Sciences, The University of Haripur, Haripur 22620, Pakistan \\ ${ }^{9}$ Department of Chemistry, University of Education Lahore, Attock campus, Attock 43600, Pakistan \\ ${ }^{10}$ Department of Chemistry, University of Swat-Swat, Mingora, Pakistan \\ ${ }^{11}$ Division of Science and Technology, Department of Chemistry, University of Education Lahore, Lahore, Pakistan
}

Published online:

17 November 2021

(C) Springer Science+Business

Media, LLC, part of Springer

Nature 2021

\section{Correction to:}

Journal of Materials Science: Materials in Electronics

https://doi.org/10.1007/s10854-021-07154-0
The original version of the article was published with errors in the name and affiliation of Dr. Ahmad Nauman Shah Saqib.

The following corrections have been made:

The original article can be found online at https://doi.org/10.1007/s10854-021-07154-0.

Address correspondence to E-mail: shahidiqbal.chem@sns.nust.edu.pk; alibahadur138@snu.ac.kr; qaiser@ccelab.com.cn 
1. The author's name incorrectly written as "Ahamd Nauma" is changed to "Ahmad Nauman Shah Saqib".

2. Affiliation of Dr. Ahmad Nauman Shah Saqib incorrectly written as "Department of Chemistry, Education University-Attock Campus (EU) Attock, Sargodha, Pakistan" is changed to "Department of Chemistry, University of
Education Lahore, Attock campus, Attock-43600, Pakistan".

The original article has been corrected.

Publisher's Note Springer Nature remains neutral with regard to jurisdictional claims in published maps and institutional affiliations. 